\title{
Operacionalidade de aplicativos de smartphone para mensuração de altura de árvores em região de ecótono Cerrado-Amazônia
}

\author{
Rafaella De Angeli CURTOํㅜㄹ Bruna Paz DEECKEN ${ }^{1}$, Sintia Valerio KOHLER ${ }^{1}$, \\ Scheila Cristina BIAZATTI ${ }^{2}$, Emanuel José Gomes de ARAÚJO ${ }^{1}$, Charlote WINK ${ }^{1}$, Rômulo MÔRA ${ }^{2}$ \\ ${ }^{1}$ Instituto de Ciências Agrárias e Ambientais, Universidade Federal de Mato Grosso, Sinop, MT, Brasil. \\ ${ }^{2}$ Faculdade de Engenharia Florestal, Universidade Federal de Mato Grosso, Cuiabá, MT, Brasil. \\ *E-mail: rafaellacurto@yahoo.com.br
}

Recebido em março/2018; Aceito em dezembro/2018.

\begin{abstract}
RESUMO: Objetivou-se verificar a operacionalidade de aplicativos de smartphones comparando-os com hipsômetro tradicional de comprovada eficiência na mensuração de altura total de árvores em fragmento florestal. O Hipsômetro Vertex IV® foi utilizado como medida real da altura total, diante da impossibilidade da obtenção da variável de forma direta. Foram selecionadas aletoriamente 90 árvores, distribuídas igualmente em três classes de altura: 1 (9,0-15,9 m); $2(16,0-22,9 \mathrm{~m})$; e 3 (>23,0 m). Para estimar a altura total foram utilizados os aplicativos para smartphones com sistema operacional Android: Two Point Height (T.P.H); Measure Height (M.H); Smart Measure (S.M) e Hypsometer (HR). Todos aplicativos, por resultar em alturas médias estatisticamente iguais a média obtida com o Vertex ${ }^{\circledR}$, podem ser indicados para a mensuração de árvores nas diferentes classes de altura. A análise residual e estatísticas Viés ( $V)$, Média das Diferenças Absolutas $(M D)$, Desvio Padrão das Diferenças $(D P D)$ e Erro Quadrático Médio (RMSE) indicaram que o Hypsometer apresentou menor tendência nas estimativas, distribuição de resíduos mais homogênea, além de resultar em menor erro médio. A atualização dos aplicativos, a resolução do acelerômetro e os cuidados na medida foram determinantes para o desempenho dos aplicativos como alternativa viável para a mensuração de árvores em relação ao custo e eficiência.
\end{abstract}

Palavras-chave: vertex, acelerômetro, custo.

\section{Operationality of smartphone applications for measuring tree height in the Cerrado-Amazon ecotone region}

\begin{abstract}
The objective verify the operability of smartphone applications comparing them with traditional hypsometer of proven efficiency in the measurement of total height of trees in forest fragment. The Vertex IV® Hypsometer was used as a real measure of total height, due to the impossibility of obtaining the variable directly. 90 trees were randomly selected, distributed equally in three height classes: 1 (9.0-15.9 m); $2(16.0-22.9 \mathrm{~m})$; and $3(>23.0 \mathrm{~m})$. To estimate total height, the following applications (Apps) were used for smartphones with Android operating system: Two Point Height (T.P.H); Measure Height (M.H); Smart Measure (S.M) and Hypsometer (HR). All tested applications, as they result in average heights statistically equal to the average obtained with the Vertex ${ }^{\circledR}$, can be indicated for the measurement of trees in the different height classes. The Bias (V), Mean of Absolute Differences (MD), Standard Deviation of Differences (DPD) and Mean Square Error (RMSE) statistics indicated, considering all applications, that the Hypsometer presented a less tendentious and more homogeneous distribution of residues, besides a smaller average error. Upgrading applications, accelerometer resolution, and tailored care were key determinants of application performance as a viable alternative for tree measurement in terms of cost and efficiency.

Keywords: vertex, acelerometer, cost.
\end{abstract}

\section{INTRODUÇÃO}

Análises florísticas e estruturais permitem obter informações sobre a situação atual de fragmentos florestais, com o intuito de estabelecer estratégias de conservação (SILVA; SOARES, 2003) orientando iniciativas de preservação e manejo de remanescentes. Para isso, se busca a mensuração de dimensões básicas como diâmetro e a altura de árvores (FELICIANO et al., 2016), necessitando de uma busca constante de alternativas visando otimizar as suas formas de obtenção.

Com os dados de altura das árvores é possível compreender a estrutura vertical da comunidade, por permitir estimar as espécies que apresentam maior importância ecológica, gerando importante significado para fins de manejo (SOUZA; SOUZA, 2004), além de permitir obter o volume de madeira de uma floresta, planejar corretamente retiradas de madeira do talhão e analisar o seu crescimento (SCOLFORO; THIERSCH, 2004). Porém, a mensuração da altura das árvores acaba se tornando uma atividade onerosa e de alto custo, de modo que se têm aumentado a busca por tecnologias e métodos mais eficientes, menos trabalhosos e que consequentemente reduza os custos das atividades do inventário florestal.

As alturas podem ser obtidas diretamente, sendo aquelas medidas tomadas sobre a árvore, ou indiretamente, baseandose em princípios geométricos e trigonométricos, cujos 
aparelhos são usualmente denominados de hipsômetros (FINGER, 2006). Entretanto, os equipamentos utilizados para a medição de alturas de árvores pressupõem a visualização da base e do topo da árvore a partir de um mesmo ponto, o que é geralmente inviável no interior das florestas tropicais (CURTO et al., 2013). Já a obtenção da altura de forma direta em florestas inequiâneas pode ser considerada não só inviável, mas em alguns casos também impraticável, devido à dificuldade e o alto custo associados a atividade, principalmente quando trata-se de florestas voltadas para a preservação, quando se deseja apenas fazer um estudo sobre a dinâmica de crescimento.

Outra limitação na obtenção da altura de árvores é a falta de recursos para a aquisição de instrumentos de medição que sejam precisos e acurados. Por isso, quando não há disponibilidade de instrumentos para medição de altura, usase a forma expedita, em que o operador se apoia em sua habilidade, a fim de obter estimativas sem nenhum instrumento (FINGER, 2006), porém tal atividade pode resultar em estimativas tendenciosas, já que depende da habilidade pessoal. Com comprovada precisão e exatidão, há no mercado aparelhos sofisticados como os da linha Vertex®, que combinam tecnologia a laser com ultrassom permitindo a medição de altura a diferentes distâncias, mesmo em vegetações densas (HAGLÖF SWEDEN AB, 2007), todavia, em comparação com outros instrumentos, a desvantagem do Vertex ${ }^{\circledR}$ é seu preço elevado (BOŽIĆ et al., 2005).

Sendo assim, dentre as diferentes formas possíveis de obter a altura de árvores, não há um consenso sobre qual a melhor em termos de eficiência (LARJAVAARA; MULLERLANDAU, 2013), economia de tempo, e que seja de baixo custo, de modo que a recomendação de cada método pode variar com as condições de cada local, e de acordo com Mayrinck et al. (2016) pode também sofrer influência da experiência do mensurador.

Buscando-se alternativas economicamente viáveis, os telefones celulares inteligentes (smartphones) aparecem como uma opção, tendo em vista que alguns modelos podem ser adquiridos com custo relativamente baixo além de apresentar certa facilidade de aprendizado até mesmo para usuários pouco experientes (GICHAMBA; LUKANDU, 2012). Em aplicações na área florestal, há uma série de aplicativos gratuitos disponíveis para smartphones, dentre os quais há aqueles desenvolvidos especificamente para a obtenção de alturas das árvores (VILLASANTE; FERNANDEZ, 2014).

As medições de altura com smartphones são feitas usando dados coletados pelo acelerômetro, que é um componente presente nos smartphones, e em alguns casos, os aparelhos mais atuais incorporam o giroscópio que aumenta a precisão dos dados obtidos. O acelerômetro é um sensor 3D capaz de detectar mudanças em aceleração linear ao longo de três eixos ortogonais, permitindo determinar o ângulo de inclinação da tela do smartphone (VILLASANTE; FERNANDEZ, 2014), e, consequentemente, repassar a informação para o aplicativo. Já o giroscópio, detecta a velocidade angular ao longo de três eixos, sendo um sensor que usa a força da gravidade identificando a posição de um objeto no espaço (VILLASANTE; FERNANDEZ, 2014), e em um smartphone detecta se o mesmo for girado no seu próprio eixo, para cima ou para baixo.

Com o auxílio da câmera e do acelerômetro presentes no telefone, os aplicativos permitem estimar a altura por meio da mensuração da distância e ângulos usando equações trigonométricas básicas (VILLASANTE; FERNANDEZ, 2014), porém há necessidade de verificar se a utilização de aplicativos disponíveis para obtenção de altura geram dados satisfatórios em termos de precisão e exatidão.

Tendo-se em vista a possibilidade do uso de smartphones, aliada a possibilidade de redução de custos nas atividades de mensuração florestal, levantamos a hipótese de que aplicativos de smartphones são exatos e podem ser utilizados para mensurar a altura de árvores. Assim, objetivou-se com o presente estudo, verificar a operacionalidade de aplicativos de smartphones comparados com hipsômetro tradicional de comprovada eficiência utilizado para mensurar a altura total das árvores, em um fragmento florestal de transição entre Cerrado e Amazônia.

\section{MATERIAL E MÉTODOS}

\section{1. Área de estudo}

O presente estudo foi realizado no Parque Florestal de Sinop, município de Sinop, Mato Grosso, localizado nas coordenadas $11^{\circ} 50^{\prime} 55^{\prime}$ 'S e $55^{\circ} 29^{\prime} 55^{\prime}$ 'O. O Parque apresenta área total de 103,98 hectares, compreendendo três reservas (R10, R11 e R12). A coleta dos dados concentrou-se na reserva $\mathrm{R} 11$, mais especificamente na área de principal visitação, em torno do lago, sendo uma Área de Preservação Permanente (APP).

De acordo com Araújo et al. (2009), devido a composição florística e estrutura, as reservas presentes no município de Sinop são classificadas como áreas de transição entre Cerrado e Floresta Amazônica, cuja vegetação é denominada de Floresta Semidecidual.

A região apresenta clima tropical, que segundo a classificação de Köppen é do tipo $A w$, caracterizado pela presença de duas estações bem definidas, sendo uma chuvosa (entre outubro e abril) e outra seca (de maio a setembro), com uma temperatura média anual de $25^{\circ} \mathrm{C}$ e a precipitação média anual de $1818 \mathrm{~mm}$ (CLIMATE-DATA, 2017).

\subsection{Coleta de dados}

Foram selecionadas aletoriamente 90 árvores, distribuídas igualmente em três classes de altura, variando de 9,0 a $15,9 \mathrm{~m}$ (classe 1), 16,0 a 22,9 m (classe 2), e acima de $23 \mathrm{~m}$ (classe 3). Para a seleção dos indivíduos foi utilizado o hipsômetro Vertex IV ${ }^{\circ}$, sendo mensurados também os respectivos diâmetros (DAP - diâmetro a 1,30 m de altura do solo) das árvores selecionadas, cuja as dimensões médias atingidas foram de 19,98 m e 31,97 cm, respectivamente.

Diante da impossibilidade para a obtenção da altura total de forma direta (escalada ou derrubada), o Hipsômetro Vertex IV® foi utilizado como medida real da altura total. Tal decisão está pautada em estudos que comprovaram sua precisão e exatidão (SILVA et al., 2012; CURTO et al., 2013; FELICIANO et al., 2016).

Para a obtenção da altura total com o Vertex IV®, inicialmente o mesmo foi devidamente calibrado. Para efetuar as medições, o transponder foi fixado a uma altura prédefinida da árvore (1,30 metros do solo), sendo possível a obtenção da distância do mensurador a árvore de forma automática, além da obtenção da altura total.

Para realização da estimação da altura com os aplicativos para smartphones, o aparelho celular utilizado foi um Motorola ${ }^{\circledR} 3^{\mathrm{a}}$ Geração, modelo XT1544TVP que contém display de cinco polegadas, e câmera de 13 megapixels. As características dos componentes foram obtidas por meio do 
aplicativo Elixir desenvolvido pela Tamás Barta (2012), no qual verificou-se que o celular possui um sensor acelerômetro de três eixos com uma resolução de $0,009576807 \mathrm{~m} \cdot \mathrm{s}^{-2}$, sendo ausente o giroscópio.

Com o acelerômetro torna-se possível verificar mudanças em aceleração linear ao longo dos três eixos ortogonais, em que o ângulo de inclinação da tela do smartphone é transformado pelo aplicativo em valores de altura, por meio do princípio trigonométrico, após obtenção da distância. O procedimento interno de cálculo é o mesmo realizado por aparelhos como o Vertex ${ }^{\circledR}$ em relação ao utilizado nos aplicativos disponíveis.

Foram utilizados para avaliação os aplicativos (app) para smartphones Two Point Height (T.P.H), Measure Height (M.H), Smart Measure (S.M) e Hypsometer (HR). Os aplicativos são disponibilizados para download de forma gratuita na plataforma Android.

Para todos os aplicativos testados, foi necessário determinar previamente a distância do mensurador até a árvore, utilizando uma trena. De modo a evitar que o mensurador fosse influenciado em relação as medidas obtidas pelos diferentes aplicativos, as avaliações foram realizadas em dias diferentes, porém, buscando-se a mesma distância entre o mensurador e a árvore para efetuar a medição.

Durante as medições, o smartphone foi mantido a uma altura de 1,68 $\mathrm{m}$ acima do solo (altura do olho do mensurador), sendo tal informação necessária ser inserida previamente nos aplicativos, exceto quando se utilizou o aplicativo Hypsometer (versão 1.11), desenvolvido pela Makinosoft (2017), em que a altura dos olhos é tomada como 1,60 m acima do solo.

Dentre os aplicativos testados, o Two Point Height (versão 0.05) desenvolvido pela Omega Centauri Software (2014), é o único que não é específico para a área florestal, em que a estimação somente é possível mirando o ponto indicado no visor do aplicativo na base e topo da

árvore, respectivamente. Com os demais aplicativos, há necessidade apenas de mirar o topo da árvore, para obter a altura total. Em todos os aplicativos as miras estão posicionadas no centro da tela.

Para estimação com Measure Height (versão 1.4), desenvolvido pela Deskis OÜ (2014), ao medir a altura há a opção de inserir uma distância do mensurador a árvore ou estimá-la com o uso do software. Com intuito de aumentar a exatidão do valor da altura, foi selecionado a opção de medir apenas a altura, sendo inserida uma distância, tendo em vista que o próprio desenvolvedor salienta que com esta opção o resultado será mais preciso.

O aplicativo Smart Measure (versão 1.6.8), desenvolvido pela Smart Tools co. (2014) apresenta a opção de corrigir a inclinação de terrenos, sendo um diferencial em relação aos demais aplicativos testados, porém, tal função não foi utilizada tendo em vista que o local apresenta terreno plano.

É importante ressaltar que, devido à ausência de um tripé ou um local de apoio para o smartphone, foi tomado o necessário cuidado para evitar oscilação do aparelho durante a leitura da altura. Dos aplicativos testados, todos dispõem de um botão na interface para estabilizar a oscilação do aparelho no momento da leitura, exceto o Hypsometer.

\subsection{Análise estatística}

Aplicou-se o teste de Bartlett a 99\% de probabilidade (SNEDECOR; COCHRAN, 1989), com intuito de verificar homogeneidade das variâncias. Atendido o pressuposto, os dados foram submetidos à análise de variância (ANOVA) em um delineamento inteiramente casualizado (DIC), e comparação de médias pelo teste de Dunnett a 99\% de probabilidade, entre os valores reais (testemunha) da altura das árvores e os valores obtidos com os diferentes aplicativos utilizados na mensuração, em cada classe de altura. As análises foram realizadas com auxílio do programa $\mathrm{R}$ ( $\mathrm{R}$ CORE TEAM, 2017).

Avaliou-se a análise gráfica de resíduos (Equação 1) com o intuito de se verificar eventuais tendências na obtenção das alturas, nas classes consideradas.

$$
\mathrm{R}=\frac{\widehat{\mathrm{Y}}_{1}-\mathrm{Y}_{\mathrm{i}}}{\mathrm{Y}_{\mathrm{i}}} \cdot 100
$$

Equação (1)

em que: $\mathrm{R}=$ Resíduo (\%); $Y_{i}$ Altura da i-ésima árvore medida com o hipsômetro Vertex ${ }^{\circledR},(\mathrm{m}) ; \hat{Y}_{i}=$ altura da i-ésima árvore medida pelos aplicativos avaliados $(\mathrm{m})$.

Também foram realizados testes complementares como as estatísticas Viés (V), Média das Diferenças Absolutas (MD), Desvio Padrão das Diferenças (DPD) e Erro Quadrático Médio (RMSE) conforme as Equações (2), (3), (4) e (5), respectivamente.

$$
\begin{gathered}
V=\frac{\sum_{i=1}^{n} Y_{i}-\sum_{i=1}^{n} \widehat{Y}_{1}}{n} \\
M D=\frac{\sum_{i=1}^{n}\left|Y_{i}-\widehat{Y}_{1}\right|}{n} \\
D P D=\sqrt{\frac{\sum_{i=1}^{n} d_{i}^{2}-\left(\frac{\sum_{i=1}^{n} d_{i}}{n}\right)^{2}}{n-1}} \\
R M S E=\sqrt{\frac{\sum_{i=1}^{n}\left(\widehat{Y}_{1}-Y_{i}\right)^{2}}{n}}
\end{gathered}
$$

em que: $\mathrm{V}=$ Viés (m); MD = Média das diferenças absolutas (m); $\mathrm{DPD}=$ Desvio padrão das diferenças $(\mathrm{m}) ; \mathrm{RMSE}=$ Erro Quadrático Médio $(\mathrm{m}) ; \mathrm{Y}_{\mathrm{i}}=$ Altura da i-ésima árvore medida com o hipsômetro Vertex ${ }^{\circledR},(m) ; \widehat{Y}_{1}=$ altura da i-ésima árvore medida pelos aplicativos avaliados $(\mathrm{m}) ; \mathrm{n}=$ número de observações; $\mathrm{d}_{\mathrm{i}}=\left(\mathrm{Y}_{\mathrm{i}}-\widehat{\mathrm{Y}}_{\mathrm{l}}\right)$.

$O$ viés indica a existência ou não de tendências entre os resíduos, porém não mede o quão afastado do eixo zero estão os valores residuais. Já a média das diferenças fornece justamente a ideia da amplitude dos erros, e o desvio padrão das diferenças mostra a homogeneidade entre os resíduos. $\mathrm{O}$ erro quadrático médio indica a diferença entre a estimativa e o verdadeiro valor da quantidade estimada, representando explicitamente o que se busca minimizar.

Os valores positivos e negativos da estatística viés indicam subestimativa e superestimativa, respectivamente, e os menores valores das quatro estatísticas testadas (V, MD, DPD e RMSE) indicam que o aplicativo para mensuração da altura apresenta maior exatidão. A partir dos resultados das estatísticas complementares foram atribuídas notas variando de 1 a 4, em que o melhor aplicativo recebeu a menor nota, de acordo com os resultados favoráveis da respectiva estatística complementar para cada classe de altura.

Todos os testes complementares tiveram seus valores relativizados (\%) visando facilitar a comparação dos 
aplicativos. Para isso, cada resultado foi dividido pela respectiva altura média das árvores medidas com o hipsômetro Vertex ${ }^{\circledR}(\mathrm{m})$.

\section{RESULTADOS}

$\mathrm{O}$ teste de Bartlett (p-valor 0,9099) indicou homogeneidade das variâncias para os dados de altura total obtidos. Na Tabela 1 estão apresentadas as médias e o p-valor para a análise de variância, considerando $99 \%$ de probabilidade obtidas para cada aplicativo, em cada classe de altura, bem como para os dados gerais.

Tabela 1. Médias das alturas (m) obtidas por classe e para os dados gerais e os respectivos p-valor para a análise de variância.

Table 1. Mean of the obtained heights $(\mathrm{m})$ per class and for the general data and the respective p-value for the analysis of variance.

\begin{tabular}{ccccc}
\multicolumn{5}{c}{ Altura média $(\mathrm{m})$} \\
\hline & Geral & Classe 1 & Classe 2 & Classe 3 \\
\hline Vertex ${ }^{\circledR}$ & 19,98 & 12,98 & 19,53 & 27,44 \\
T.P.H & 21,34 & 13,81 & 21,12 & 29,10 \\
M.H & 19,87 & 12,57 & 19,96 & 27,07 \\
S.M & 20,80 & 13,17 & 21,30 & 27,95 \\
HR & 19,71 & 12,20 & 20,14 & 26,78 \\
\hline p-valor & $0,4634^{\mathrm{ns}}$ & $0,0803^{\mathrm{ns}}$ & $0,0257^{\mathrm{ns}}$ & $0,4543^{\mathrm{ns}}$ \\
\hline
\end{tabular}

em que: * = diferença significativa $(\mathrm{p}<0,01) ; \mathrm{ns}=$ diferença não significativa $(\mathrm{p}>0,01)$ para o teste $\mathrm{F}$.

A partir do p-valor (Tabela 1) verificou-se que não houve diferença significativa entre as médias de altura gerais como por classe de altura, obtidos pelos diferentes aplicativos testados, não necessitando da realização do teste de Dunnet.

Em complemento, é apresentada uma análise gráfica dos resíduos (Figura 1) levando-se em conta os aplicativos avaliados nas diferentes classes de altura.

Nota-se que os aplicativos Two Point Height (T.P.H) e Smart Measure (S.M) apresentaram evidente tendência em superestimar a altura em todas as classes avaliadas, já os aplicativos Measure Height (M.H) e Hypsometer (HR) apresentaram tal tendência apenas na classe 2 de altura. Para as demais classes, de um modo geral, os aplicativos Measure Height e Hypsometer apresentaram leves tendências em subestimar a altura total. Apesar da observação dessas tendências, no geral, a amplitude dos erros ocasionados com a mensuração baseada em aplicativos de smartphone foi baixa. No entanto, o aplicativo Hypsometer apresentou distribuição mais uniforme em todas as classes.

As estatísticas Viés (V), Média das Diferenças Absolutas (MD), Desvio Padrão das Diferenças (DPD) e Erro Quadrático Médio (RMSE) para as estimativas de altura, pelos diferentes aplicativos avaliados, para as diferentes classes consideradas podem ser observadas na Tabela 2 .

Analisando-se os resultados mostrados na Tabela 2, ao considerar as estatísticas complementares por classe, verificase que o Two Point Height teve melhor desempenho para mensurar árvores da classe 1, porém, a medida que a altura aumentou, sua eficiência reduziu. Já o aplicativo Smart Measure, se mostrou mais eficiente para mensurar árvores da classe $3(>23,0 \mathrm{~m})$. O Measure Height e Hypsometer, conforme também verificado na análise de resíduos (Figura 1) foram mais eficientes para mensurar a altura de árvores na classe intermediária (entre 16,0 e 23,0 m).

O Hypsometer foi o aplicativo que resultou em menor nota (Tabela 2), considerando todas as classes de altura conjuntamente, indicando que de um modo geral, apresentou distribuição de resíduos menos tendenciosa (V) e mais homogênea (DPD), menor erro médio (MD), e menor erro quadrático médio (RMSE), indicando menor diferença entre a estimativa e o verdadeiro valor da quantidade estimada. Porém, observando de forma geral, embora o Hypsometer tenha obtido a menor nota, o seu melhor desempenho pode ser alterado em função da classe de altura. Os outros três aplicativos apresentaram diferença relativamente pequena entre si.

Tabela 2. Estatísticas Viés (V), Média das Diferenças Absolutas (MD), Desvio Padrão das Diferenças (DPD) e Erro Quadrático Médio (RMSE) em porcentagem, para as estimativas de altura total nas diferentes classes, obtidas com aplicativos de smartphone, com as respectivas notas atribuídas com base nas estatísticas.

Table 2. Statistics Bias (V), Mean Absolute Differences (MD), Standard Deviation of Differences (DPD) and Mean Square Error (RMSE), in percentage, for total height estimates obtained with smartphone app, with the respective scores assigned based on statistics.

\begin{tabular}{|c|c|c|c|c|c|c|c|c|c|}
\hline & & T.P.H & & M.H & & S.M & & HR & \\
\hline & $\bar{V}$ & $-6,37$ & 4 & 3,13 & 2 & $-1,46$ & 1 & 5,99 & 3 \\
\hline \multirow{5}{*}{ Classe 1} & $M D$ & 9,60 & 1 & 11,66 & 4 & 10,35 & 2 & 10,73 & 3 \\
\hline & $D P D$ & 12,95 & 1 & 14,23 & 3 & 14,72 & 4 & 13,39 & 2 \\
\hline & RMSE & 12,78 & 1 & 14,00 & 3 & 14,48 & 4 & 13,21 & 2 \\
\hline & Total & & 7 & & 12 & & 11 & & 10 \\
\hline & $V$ & $-8,11$ & 3 & $-2,17$ & 1 & $-9,03$ & 4 & $-3,11$ & 2 \\
\hline \multirow[t]{5}{*}{ Classe 2} & $M D$ & 11,41 & 3 & 11,28 & 2 & 12,13 & 4 & 9,77 & 1 \\
\hline & $D P D$ & 15,61 & 4 & 14,20 & 2 & 15,11 & 3 & 12,00 & 1 \\
\hline & RMSE & 15,42 & 4 & 13,96 & 2 & 14,95 & 3 & 11,81 & 1 \\
\hline & Total & & 14 & & 7 & & 14 & & 5 \\
\hline & $V$ & $-8,49$ & 4 & 1,88 & 1 & $-2,59$ & 2 & 3,38 & 3 \\
\hline \multirow[t]{5}{*}{ Classe 3} & $M D$ & 12,11 & 1 & 14,78 & 4 & 12,97 & 2 & 14,60 & 3 \\
\hline & $D P D$ & 17,25 & 3 & 18,57 & 4 & 17,09 & 1 & 17,23 & 2 \\
\hline & RMSE & 12,12 & 3 & 13,00 & 4 & 11,97 & 1 & 12,07 & 2 \\
\hline & Total & & 11 & & 13 & & 6 & & 10 \\
\hline & Total Geral & & 32 & & 32 & & 31 & & 25 \\
\hline
\end{tabular}


Classe $1(9,0$ a $15,9 \mathrm{~m})$
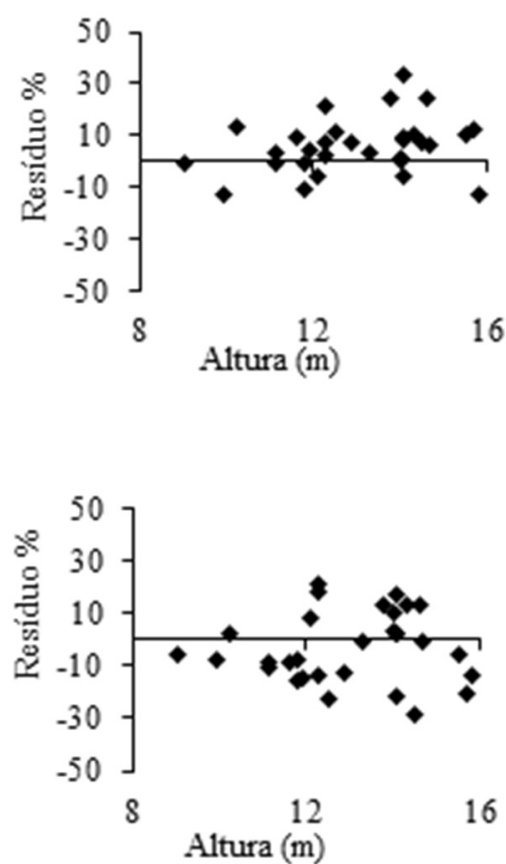

Classe 2 (16,0 a 22,9 m)

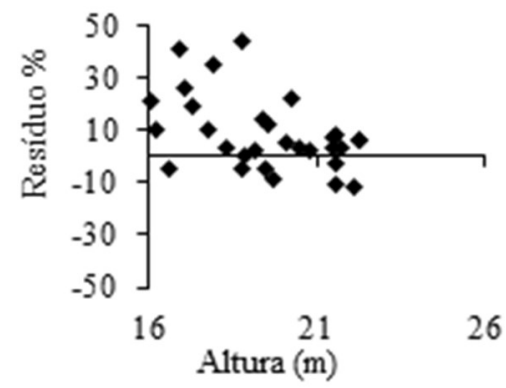

Measure Height (M.H)

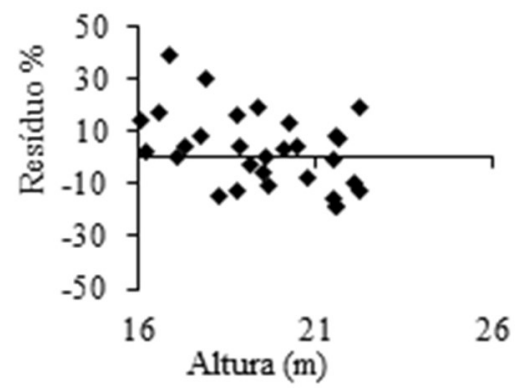

Smart Measure (S.M)
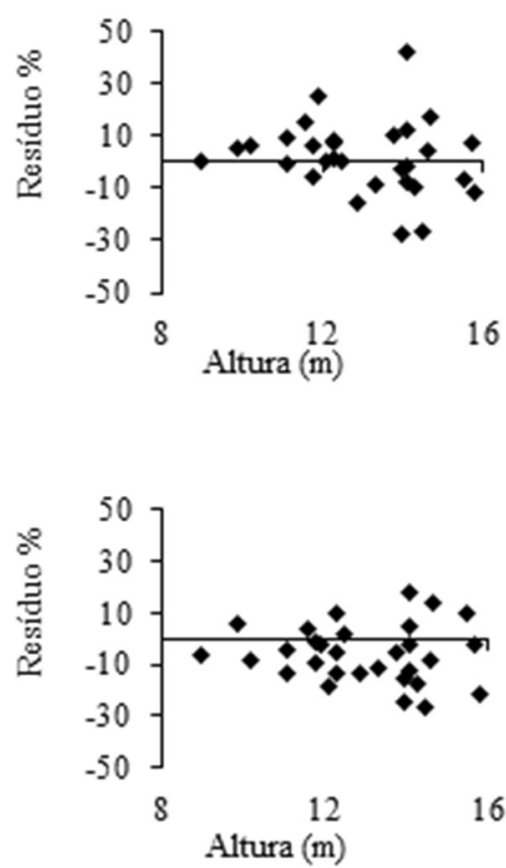

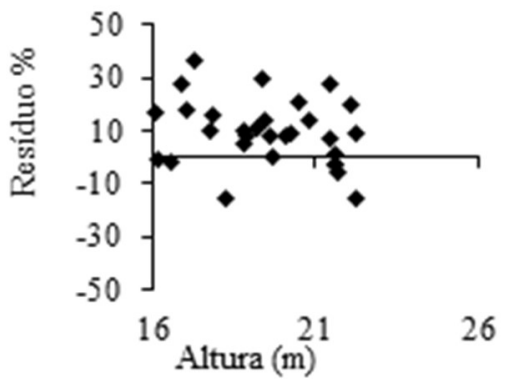

Hypsometer (HR)

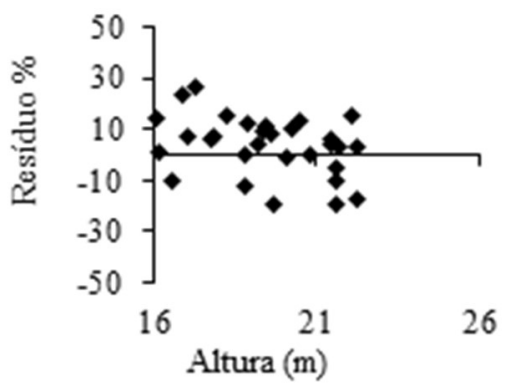

Classe $3(>23,0 \mathrm{~m})$
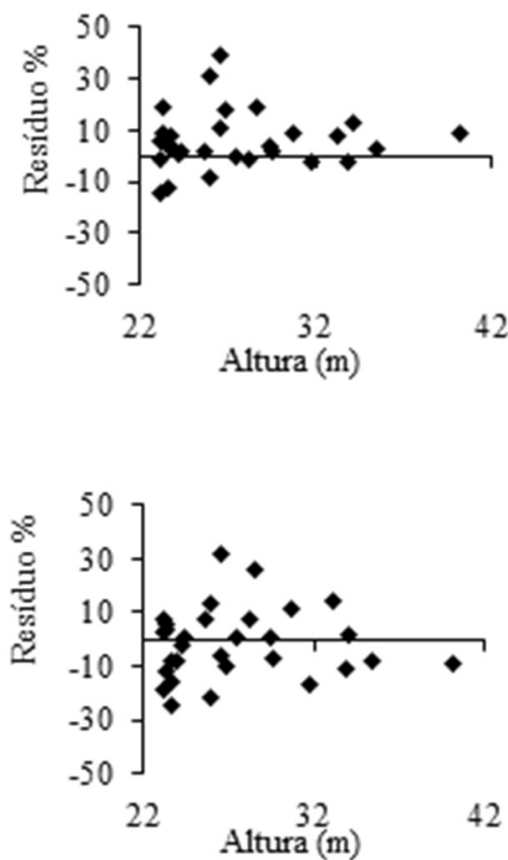

Figura 1. Distribuição dos resíduos de altura, em percentagem, para quatro aplicativos analisados, em três classes de altura. Figure 1. Distribution of height residues, in percentage, for the four analyzed apps, for the three height classes evaluate.

\section{DISCUSSÃO}

O Vertex ${ }^{\circledR}$ é um equipamento tradicional da área florestal, de comprovada eficiência, podendo-se citar trabalhos desenvolvidos por Silva et al. (2012) e Curto et al. (2013), que verificaram igualdade estatística entre a altura real obtida com escalada e a altura obtida com o Vertex® em florestas nativas, considerando diferentes amplitudes de altura. Adicionalmente, Feliciano et al. (2016) verificaram alta precisão do Vertex ${ }^{\circledR}$ quando comparado a outros seis tipos de hipsômetros. O Vertex ${ }^{\circledR}$ foi utilizado também por Villasante; Fernandez (2014), atestando a eficiência deste equipamento.
Todos aplicativos testados, por resultar em altura média estatisticamente iguais a média obtida com o Vertex ${ }^{\circledR}$ a princípio podem ser indicados para a mensuração de árvores com alturas variando desde as mais baixas (Classe 1 - a partir de 9,0 $\mathrm{m}$ ) até as mais altas (Classe $3-<23,0 \mathrm{~m}$ ).

Villasante; Fernandez (2014), também testando os aplicativos Smart Measure e Measure Height, afirmam que não houve igualdade das alturas médias quando comparadas com as medidas obtidas pelo Vertex ${ }^{\circledR}$. Conforme esses autores, uma explicação para este resultado é que os aplicativos possuíam uma transformação interna deficiente 
entre os dados obtidos pelo acelerômetro e sua conversão em valores de ângulo e consequentemente em altura, sugerindo incorporar uma calibração do acelerômetro como solução para tal deficiência em todos os aplicativos de smartphones destinados a estimar os ângulos com base nas medidas do acelerômetro.

É importante ressaltar que os aplicativos utilizados por Villasante; Fernandez (2014) são versões desatualizadas, sendo o Smart Measure (versão 1.5.0) e Measure Height (versão 1.3), ambas de 2012. No presente trabalho, foram utilizadas as versões do Smart Measure (versão 1.6.8) de 2017 e Measure Height (versão 1.4) de 2014. Assim, acredita-se que com a atualização os erros possam ter sido corrigidos, resultando em melhor desempenho dos aplicativos.

Para os demais aplicativos, não foram encontrados trabalhos específicos para averiguar sua exatidão, porém ambos possuem versões recentes, sendo o Two Point Height (versão 0.05) de 2014 e Hypsometer (versão 1.11) de 2017. É importante destacar que, diferente dos demais aplicativos, o Two Point Height, desenvolvido pela Omega Centauri Software, não é um aplicativo específico da área florestal.

$\mathrm{Na}$ tentativa de contornar os erros em subestimar os valores verdadeiros detectados pelo uso de aplicativos, dada a linearidade mantida por tais erros, Villasante; Fernandez (2014), efetuaram uma correção usando regressão linear, resultando em uma considerável melhoria nas estimativas, porém, ainda assim foram detectados erros sistemáticos para os dois aplicativos. Esses erros foram menores que os encontrados por Bijak; Sarzyński (2015) quando testados os mesmos aplicativos em versões mais atuais (ambos de 2014), em que após realizarem correções, ambos tiveram tendência de subestimar a altura da árvore, independente da distância do mensurador a árvore no momento da medição, com destaque negativo para o Measure Height, que produziu maiores erros que o Smart Measure, principalmente para árvores mais altas.

Vale ressaltar que Villasante; Fernandez (2014) tomaram medidas para pontos pré-definidos de altura entre 6 e $12 \mathrm{~m}$, eliminando o problema de identificação e localização das árvores, reduzindo os erros. Todavia, Bijak; Sarzyński (2015) analisaram árvores em condições semelhantes ao presente estudo, onde é mais difícil localizar o topo da árvore, além das alturas das árvores terem sido maiores, variando entre 12,0 e 29,0 m. Assim, uma razão para os erros terem sido maiores, deve-se a maior dificuldade de visualização e maior altura das árvores. Até porque, Bijak; Sarzyński (2015), em ambos os aplicativos testados, verificaram exatidão na estimação da altura de árvores de menor porte (12,0-18,0 m), no entanto, houve subestimativa para árvores mais altas $(>20,0 \mathrm{~m})$.

Pela análise de resíduo (Figura 1) não se nota um padrão de tendências regular com o aumento da altura, já que os aplicativos Measure Height e o Hypsometer superestimaram as alturas da classe intermediária, porém apresentaram tendência em subestimar as alturas de forma mais acentuada nas classes inferior e superior. As classes de altura foram estabelecidas justamente para captar uma possível perda de exatidão e crescimento da tendência com o aumento da altura. Bijak; Sarzyński (2015), com o uso de aplicativos verificaram que as tendências aumentam com o aumento da altura das árvores. Curto et al. (2013) e Silva et al. (2012) também verificaram essa tendência com o uso de hipsômetros tradicionais na área florestal.

De acordo com Itoh et al. (2010) a tendência seria que a exatidão caísse à medida que a altura e o ângulo de elevação aumentassem, porém não foi um padrão observado no presente estudo, pois cada classe de altura se comportou de forma diferente dependendo do aplicativo. Deste modo, não seria somente a classe de altura responsável pela redução da exatidão, mas também o ângulo de inclinação do smartphone, o que está diretamente relacionado a distância em que o mensurador se posiciona em relação a árvore. Porém, buscouse um afastamento sempre superior ao que se imaginava ser a altura da árvore, desde que possível visualizar seu topo. No entanto, não há como garantir que as distâncias realmente tenham sido superiores, uma vez que a altura ainda seria obtida. Assim, com relação aos aplicativos, uma vez que utilizam a inclinação do smartphone e os cálculos se baseiam no princípio trigonométrico para a determinação da altura da árvore, um pequeno desvio da vertical pode resultar em um erro significativo (BIJAK; SARZYŃSKI, 2015).

De acordo com Silva et al. (2017), além da altura da árvore e distância reduzida do mensurador até a base da árvore, o ângulo de inclinação da árvore e o ângulo de inclinação do terreno afetam o desempenho dos aparelhos baseados em princípios trigonométricos na estimação da altura. $\mathrm{O}$ ângulo de inclinação da árvore não foi avaliado no presente estudo, não sendo possível gerar uma discussão ampliada sobre este assunto. Além de que, o presente trabalho foi desenvolvido em área plana, e não seria a inclinação do terreno a responsável pela falta de um padrão com relação as tendências ao longo das classes. De todo modo, se fosse o caso, haveria necessidade de correção da inclinação do terreno.

Outro fator a ser considerado é a densidade da floresta, em que no presente estudo, por ser tratar de uma área de visitação com recorrente interferência antrópica, apresenta-se pouco densa, o que pode estar relacionado ao desempenho satisfatório dos aplicativos, visto que há uma maior dificuldade de visualização do topo da árvore em ambientes de alta densidade.

O desempenho satisfatório dos aplicativos utilizados quando comparados aos testes realizados por outros trabalhos como o de Villasante; Fernandez (2014) e Bijak; Sarzyński (2015), além de relacionados com a atualização do software, pode ser devido a resolução do acelerômetro, segundo levantado pelos próprios autores. Villasante; Fernandez (2014), utilizando um smartphone Samsung Galaxy Note, verificaram erros maiores que os observados no presente estudo, sendo a resolução do acelerômetro de $0,0383 \mathrm{~m} \cdot \mathrm{s}^{-2}$, mesmo contando com a presença de um giroscópio. Ao utilizar um modelo de smartphone com resolução do acelerômetro de $0,153 \mathrm{~m} . \mathrm{s}^{-2}$, sem a presença de giroscópio, verificou erros ainda maiores. Embora o Motorola $3^{\mathrm{a}}$ Geração utilizado não possua giroscópio, o acelerômetro possui resolução superior $\left(0,0095 \mathrm{~m} \cdot \mathrm{s}^{-2}\right)$.

Além dos fatores considerados, os resultados podem ter sofrido influência com relação ao treinamento do mensurador. Conforme estudo feito por Mayrinck et al. (2016) para cada operador, os aparelhos se comportam diferentemente, indicando que cada um se adapta mais facilmente com determinados aparelhos, influenciando no erro final. Assim, ressalta-se que todas as medições foram realizadas pelo mesmo operador, estando ele apto a operar cada aplicativo de acordo com suas configurações específicas, realizando os ajustes e calibração requerida para cada aplicativo de acordo com indicações do desenvolvedor.

Acredita-se que o melhor desempenho do aplicativo Hypsometer possa estar relacionado à leitura da altura 
diretamente no visor do smartphone sem necessidade de pressionar algum botão ao realizar a medição como ocorreu com os demais aplicativos. Porém, uma desvantagem desse aplicativo é o fato de ser necessário memorizar a altura obtida para posterior anotação, já que o mesmo não registra a informação no visor.

Apesar do Vertex ${ }^{\circledR}$ permitir a obtenção da altura de forma automática, e com os aplicativos ser necessário obter a distância utilizando uma trena, sendo comprovada a qualidade das medições com um smartphone e tendo em vista que preço do smartphone utilizado no presente estudo é até 10 vezes menor que o preço de um hipsômetro Vertex ${ }^{\circledR}$, os aplicativos gratuitos para smartphones tornam-se uma opção viável para a mensuração da altura total de árvores, desde as de menor até maior porte.

Porém, ressalta-se que devem ser observadas as condições de densidade da floresta e de inclinação do terreno, distância entre o mensurador e a árvore, e consequentemente o ângulo de inclinação do smartphone, além do treinamento do mensurador, e características do celular, como a resolução do acelerômetro.

\section{CONCLUSÕES}

Aplicativos para smartphones foram eficientes para obter a altura total de árvores, em variadas classes de altura, resultando em igualdade estatística com as medidas de um hipsômetro tradicional de comprovada eficiência na área florestal.

O aplicativo Hypsometer apresentou melhor desempenho com distribuição de resíduos menos tendenciosa e mais homogênea, além de menor erro médio. Diferencia-se dos demais em termos práticos, pela sua recente versão e por não necessitar de acionamento de botão ao realizar a medição, tendo-se a leitura da altura diretamente no visor do smartphone.

A atualização dos aplicativos, a resolução do acelerômetro e os cuidados na medida são fatores determinantes para o bom desempenho dos aplicativos, sendo uma alternativa para a mensuração de árvores quando se deseja exatidão e custos baixos.

\section{REFERÊNCIAS}

ARAÚJO, R. de A.; COSTA, R. B. da; FELFILI, J. M.; KUNTZ, I. G.; SOUZA, R. A. T. de M. e; DORVAL, A. Florística e estrutura de fragmento florestal em área de transição na Amazônia Matogrossense no município de Sinop. Acta Amazônica, Manaus, v. 39, n. 4, p. 865-878, $2009 . \quad$ DOI: http://dx.doi.org/10.1590/S004459672009000400015

BIJAK, S.; SARZYŃSKI, J. Accuracy of smartphone applications in the field measurements of tree height. Folia Forestalia Polonica, Raszyn, v. 57, n. 4, p. 240-244, 2015. DOI: https://dx.doi.org/10.1515/ffp-2015-0025

BOŽIĆ, M.; ĆAVLOVIĆ, J., LUKIĆ, N.; TESLAK, K.; KOS, D. Efficiency of ultrasonic Vertex III hypsometer compared to the most commonly used hypsometers in Croatian forestry. Croatian Journal of Forest Engineering, Zagreb, v. 26, n. 2, p. 91-99, 2005.

CLIMATE-DATA. Clima: Sinop. Disponível em: $<$ http://pt.climate-data.org/location/4077/> Acesso em: 12 mar 2018.

CURTO, R. de A.; SILVA, G. F. da.; SOARES, C. P. B.; MARTINS, L. T.; DAVID, H. C. Métodos de estimação de altura de árvores em Floresta Estacional Semidecidual. Revista Floresta, Curitiba, v. 43, n. 1, p. 105-116, jan./mar. 2013.

DOI: http://dx.doi.org/10.5380/rf.v43i1.26791

DESKIS OU. Measure Height. 2014. Disponível em: $<$ https://play.google.com/store/apps/details?id=ee.deskis.a ndroid.height\&hl=en>. Acesso em: 12 mar 2018.

FELICIANO, M. E.; RIBEIRO, A.; FERRAZ FILHO, A. C.; VITOR, P. C. G. Avaliação de diferentes hipsômetros na estimativa da altura total. Revista Verde de Agroecologia e Desenvolvimento Sustentável, v. 11, n. 2, p. 01-05, abr./jun. 2016.2 DOI: http://dx.doi.org/10.18378/rvads.v11i2.3741

FINGER, C. A. G. Biometria Florestal. Santa Maria: Departamento de Ciências Florestais, 2006. 314 p.

GICHAMBA, A.; LUKANDU, I. A. A model for designing $\mathrm{m}$-agriculture applications for dairy farming. The African Journal of Information Systems, v. 4, n. 4, 2012.

HAGLÖF SWEDEN AB. Users Guide Vertex IV and Transponder T3. v. 10, 2007. Disponível em: < http://www.haglof.jp/download/vertex_iv_me.pdf> Acesso em: 12 mar 2018.

ITOH T.; EIZAWA J.; YANO N.; MATSUE K.; NAITO K. Development of software to measure tree Heights on the smartphone. Journal of the Japanese Forest Society, v. 92, n. 4, p. 221-225. 2010. DOI: http://dx.doi.org/10.4005/jjfs.92.221

LARJAVAARA, M.; MULLER-LANDAU, H. C. Measuring tree height: a quantitative comparison of two common field methods in a moist tropical forest. Methods in Ecology and Evolution, London, v. 4, n. 9; p. 793-801, 2013. DOI: http://dx.doi.org/10.1111/2041-210X.12071

MAKINOSOFT. Hypsometer. 2017. Disponível em: $<$ https://play.google.com/store/apps/details?id=makino.an droid.hypsometer\&hl=pt_BR>. Acesso em: 12 mar 2018.

MAYRINCK, R. C.; OLIVEIRA, X. M.; SILVA, G. C. C.; VITOR, P. C. G.; FERRAZ FILHO, A. C. Avaliação de hipsômetros e operadores na mensuração de árvores de Eucalyptus urograndis de tamanhos diferentes. Revista Verde de Agroecologia e Desenvolvimento Sustentável, v. $11, \quad$ n. 5 , p. 90-94. 2016. DOI: http://dx.doi.org/10.18378/rvads.v11i5.3976

OMEGA CEnTAUri SOFTWARE. Two Point Height. 2014. Disponível em: $<$ https://play.google.com/store/apps/details?id=mobi.ome gacentauri.twopoint $>$. Acesso em: 12 mar 2018.

R DEVELOPMENT CORE TEAM. R: A language and environment for statistical computing. $R$ Foundation for Statistical Computing, Vienna, Austria, 2017. Disponível em: <http://www.R-project.org/>.

SCOLFORO, J. R. S.; THIERSCH, C. R. Biometria Florestal: medição, volumetria e gravimetria. Lavras: Universidade Federal de Lavras, 2004. 285 p.

SILVA, G. F. da; CURTO, R. de A.; SOARES, C. P. B.; PIASSI, L. de C. Avaliação de métodos de medição de altura em florestas naturais. Revista Árvore, Viçosa, v. 36, n. 2, p. 341-348, 2012. DOI: http://dx.doi.org/10.1590/S0100-67622012000200015

SILVA, G. F. da; MÔRA, R.; CURTO, R. de A. Simulação de erros na medição de altura de árvores inclinadas com aparelhos baseados em princípios trigonométricos. Revista Nativa, Sinop, v. 5, n. 5, p. 372-379, set./out., 2017. DOI: http://dx.doi.org/10.5935/2318-7670.v05n05a12 
Curto et al.

SILVA, L. A.; SOARES, J. J. Composição florística de um fragmento de floresta estacional semidecídua no município de São Carlos-SP. Revista Árvore, Viçosa, v. 27, n. 5, p.647-656, 2003. DOI: http://dx.doi.org/10.1590/S010067622003000500007

SMART TOOLS CO. Telêmetro: Smart Measure. 2014. Disponível

em:

$<$ https://play.google.com/store/apps/details?id=kr.sira.me asure\&hl=pt_BR $>$. Acesso em: 12 mar 2018.

SNEDECOR, G. W.; COCHRAN, W. G. Statistical Methods 8. ed. Lowa: lowa State University Press, 1989. p. 247.
SOUZA D. R.; SOUZA, A. L. Estratificação vertical em floresta ombrófila densa de terra firme não explorada, Amazônia Oriental. Revista Árvore, Viçosa, v. 28, n. 5, p. 691-698, 2004. DOI: http://dx.doi.org/10.1590/S010067622004000500008

TAMÁS BARTA. Elixir. 2012. Disponível em: $<$ https://play.google.com/store/apps/details?id=bt.android. elixir> Acesso em: 10 nov 2017.

VILLASANTE, A.; FERNANDEZ, C. Measurement errors in the use of smartphones as low-cost forestry hypsometers. Silva Fennica, Helsinki, v. 48, n. 5, p. 1-11, 2014. DOI: http://dx.doi.org/10.14214/sf.1 